\title{
A Direct Descending Pathway Informing Locomotor Networks about Tactile Sensor Movement
}

\author{
미an M. Ache, ${ }^{1,2}$ S. Shuichi Haupt, ${ }^{1}$ and $\odot$ Volker Dürrr ${ }^{1,2}$ \\ ${ }^{1}$ Department of Biological Cybernetics, Faculty of Biology, Bielefeld University, 33615 Bielefeld, Germany, and ${ }^{2}$ Cognitive Interaction Technology Center of \\ Excellence, Bielefeld University, 33615 Bielefeld, Germany
}

Much like visually impaired humans use a white-cane, nocturnal insects and mammals use antennae or whiskers for near-range orientation. Stick insects, for example, rely heavily on antennal tactile cues to find footholds and detect obstacles. Antennal contacts can even induce aimed reaching movements. Because tactile sensors are essentially one-dimensional, they must be moved to probe the surrounding space. Sensor movement is thus an essential cue for tactile sensing, which needs to be integrated by thoracic networks for generating appropriate adaptive leg movements. Based on single and double recordings, we describe a descending neural pathway comprising three identified $\mathrm{ON}$ - and OFF-type neurons that convey complementary, unambiguous, and short-latency information about antennal movement to thoracic networks in the stick insect. The neurons are sensitive to the velocity of antennal movements across the entire range covered by natural movements, regardless of movement direction and joint angle. Intriguingly, none of them originates from the brain. Instead, they descend from the gnathal ganglion and receive input from antennal mechanoreceptors in this lower region of the CNS. From there, they convey information about antennal movement to the thorax. One of the descending neurons, which is additionally sensitive to substrate vibration, feeds this information back to the brain via an ascending branch. We conclude that descending interneurons with complementary tuning characteristics, gains, input and output regions convey detailed information about antennal movement to thoracic networks. This pathway bypasses higher processing centers in the brain and thus constitutes a shortcut between tactile sensors on the head and the thorax.

Key words: tactile sensing; descending control; identified neurons; adaptive locomotion; invertebrates

\section{Introduction}

Many animals rely on their tactile sense for near-range orientation, including mammals (Diamond et al., 2008) and insects (Staudacher et al., 2005). Rats and mice, for example, navigate labyrinths (Vincent, 1912), cross gaps (Hutson and Masterton, 1986), and localize objects (Knutsen et al., 2006) based on whisker-mediated tactile cues. Similarly, insects follow walls (Camhi and Johnson, 1999), cross gaps (Bläsing and Cruse, 2004), negotiate obstacles (Harley et al., 2009), and turn toward objects (Okada and Toh, 2000) based on antennal tactile cues. For all these behaviors, information from tactile sensors must be mediated to thoracic motor networks to drive adaptive motor responses.

Tactile probes, such as antennae or whiskers, are essentially one-dimensional structures and must be moved to sample a 3D

\footnotetext{
Received Aug. 12, 2014; revised Jan. 11, 2015; accepted Jan. 24, 2015.

Author contributions: J.M.A. and V.D. designed research; J.M.A. and S.S.H. performed research; J.M.A. analyzed data; J.M.A. and V.D. wrote the paper.

This work was supported by Cluster of Excellence 277 Cognitive Interaction Technology Center of Excellence Project SP10, funded through Excellence Initiative of the German Research Council. We thank Tom Matheson for comments on an earlier version of the manuscript.

The authors declare no competing financial interests.

Correspondence should be addressed to either Jan M. Ache or Dr. Volker Dürr, Department of Biological Cybernetics, Faculty of Biology, Bielefeld University, Universitätsstr. 25, 33615 Bielefeld, Germany. E-mail: jan.m.ache@gmail.com or volker.duerr@uni-bielefeld.de.

DOI:10.1523/JNEUROSCI.3350-14.2015

Copyright $\odot 2015$ the authors $\quad 0270-6474 / 15 / 354081-11 \$ 15.00 / 0$
}

space. Because sensor movement is obstructed by obstacle contact, changes in movement can indicate contact events. Moreover, external forces can deflect tactile sensors passively, for example, when animals encounter an obstacle (Zurek and Gilbert, 2014) or an approaching predator (Comer et al., 2003). Hence, monitoring sensor movement is crucial for making sense of tactually acquired information.

Nocturnal, canopy-dwelling stick insects rely heavily on their tactile sense and constantly sample the ambient space by actively moving their antennae (Dürr et al., 2001). Antennal contact can initiate climbing by triggering an aimed front leg movement with short delay (Schütz and Dürr, 2011). These movements are similar to aimed reaching movements in vertebrates (Esposito et al., 2014). Touch-induced reaching movements require the fast transfer of mechanosensory information from the antennae to thoracic motor networks. Specifically, descending interneurons (DINs) need to transfer information about antennal movement, posture, and contact site to front leg motor networks in the prothoracic ganglion.

In several arthropods, DINs have been identified that drive distinct behavioral patterns, such as singing (Hedwig, 1994) or changes in walking direction (Bidaye et al., 2014). As yet, their upstream input organization remains elusive. Other DINs mediate antennal mechanosensory information to the thorax. They encode the occurrence of antennal contact events (Burdohan and Comer, 1996; Schöneich et al., 2011), as well as antennal pointing 
direction and movement (Gebhardt and Honegger, 2001). Because of differences among species or experimental conditions, existing data about descending information associated with tactile sensing remain fragmentary, as they do not explain how multiple parts of the same descending pathway act together to mediate fast, goal-directed behavior.

We used the stick insect, in which both tactile sensory (Dürr, 2014) and thoracic motor systems (Büschges, 2012) are well described, to study how thoracic neural networks are informed about tactile sensor movement. In an earlier study, we characterized a large population of DINs that encoded antennal posture, movement, and movement velocity (Ache and Dürr, 2013). The number and identity of these neurons, the relevant sensory structures, and their projection sites, however, were not analyzed.

Here, we identify and characterize a set of three velocitysensitive DINs. We show that they encode complementary information. Using double recordings, we analyze how this triplet of neurons jointly mediates precise information about the movement of both antennae to the thorax.

\section{Materials and Methods}

Animal preparation and stimulation. Adult, female stick insects (Carausius morosus) were fixed ventral side down on an experimental platform, after ablation of all legs. The stick insect antenna features two hinge joints with a single degree of freedom each. The head-scape joint of the right antenna was firmly immobilized with dental glue (Protemp, 3M ESPE), and the right flagellum was ablated at the level of its fifth antennomere, to preserve only the stiff flagellar base (Dirks and Dürr, 2011). The tip of a metal minutien pin was inserted into the stump of the flagellum, such that the right antenna could be moved about the scape-pedicel (SP) joint using a contact-free magnetic stimulator. It was thus possible to impose controlled SP joint movements without touching the antenna and thereby stimulating contact-sensitive mechanoreceptors. The SP joint rotation axis is slanted by $28^{\circ}$ with respect to the horizontal plane, so that levation of the joint is accompanied by adduction and depression by abduction (Dürr et al., 2001). The stimulator moved the SP joint about its natural rotation axis. The stimulation covered an angular range of $104^{\circ}$, slightly exceeding the actively used working range of the SP joint, which is $100^{\circ}$ (Krause et al., 2013). The stimulator was either driven using staircase functions, with ramps covering velocities between 1 and $800 \%$, or using sinusoids with frequencies between 0.001 and $2.5 \mathrm{~Hz}$. Thus, the stimuli covered the entire amplitude and velocity range of naturally occurring movements.

To reliably elicit spikes in the contralateral ON-type velocity-sensitive DIN (cONv), vibration stimuli were delivered directly to the recording table, using a Ling vibrator (model V201, Ling Dynamic Systems).

Electrophysiological recordings. Extracellular whole-nerve recordings from connectives at different levels of the CNS were obtained with unipolar tungsten hook electrodes. For intracellular recordings, DIN axons were impaled in the neck connectives, just anterior to the prothoracic ganglion. Electrodes were pulled from thin-walled borosilicate glass capillaries and filled with 5\% Neurobiotin (tip, Vector Labs) and $1 \mathrm{~m} \mathrm{KCl}$ (shaft), or $5 \%$ Lucifer yellow (tip) and $1 \mathrm{M} \mathrm{LiCl}$ (shaft). The electrodes had tip resistances between 20 and $60 \mathrm{M} \Omega$. For a more detailed description of the animal preparation and antennal stimulation, see Ache and Dürr (2013).

Morphology of descending interneurons. After physiological characterization, DINs were injected with Neurobiotin or Lucifer yellow, using pulsed positive or negative current, respectively, for 15-90 min, with amplitudes between 1 and $5 \mathrm{nA}$. After tracer injection, the connectives posterior to the prothoracic ganglion were crushed, and the tracer was left to diffuse between the prothoracic ganglion and the brain for up to $24 \mathrm{~h}$ at room temperature. Afterward, the brain, gnathal ganglion, and prothoracic ganglion were dissected out, fixed overnight at $4^{\circ} \mathrm{C}$ in RotiHistofix (Carl Roth), dehydrated in an ascending ethanol series, and cleared in methyl salicylate (Carl Roth). Neurobiotin was visualized after rehydration using Cy-3-Streptavidin (Jackson ImmunoResearch Labo- ratories) or CF633-Streptavidin (Biotium) by incubation for 12-24 h at 1:100 dilution in Sørensen's phosphate buffer $(0.1 \mathrm{M})$ containing $0.5 \%$ Triton-X and 3\% normal goat serum. Chemicals were obtained from Sigma-Aldrich unless noted otherwise.

Anterograde labeling of SP joint hair field afferents was achieved by incubating clipped hair field bristles in $1 \%$ tetramethylrhodamine dextran (D-3308, Invitrogen) in water for at least $24 \mathrm{~h}$ at room temperature before starting DIN recordings.

Individual ganglia mounted in methyl salicylate were scanned using a Leica SP2 confocal laser-scanning microscope (Leica Microsystems) with $20 \times$ and $40 \times$ oil-immersion objectives. Maximum projections of identified DINs were generated in ImageJ (National Institutes of Health, Bethesda, MD).

Data were analyzed using custom-written MATLAB scripts (version 7.9, MathWorks).

\section{Results}

We probed the CNS of the stick insect (C. morosus) for neurons conveying information about antennal movement to thoracic neural networks. Three individual DINs that responded to imposed antennal movements in a velocity-dependent manner were characterized and anatomically identified by intracellular recordings and tracer injection (Fig. 1). These DINs belong to a larger population of neurons mediating information about antennal movement, position, and touch events as well (Ache and Dürr, 2013). Velocity-sensitive DINs were sensitive to SP joint movement across a wide range of velocities, and their activity was largely independent of the antennal joint angle and the direction of antennal movement. Using sharp microelectrodes, each DIN was recorded in the neck connectives of at least 10 animals. The antenna was stimulated in a behaviorally relevant way, covering the entire joint angle work range and a wide range of naturally occurring movement velocities at the SP joint.

\section{Velocity-sensitive DINs have complementary input and output patterns}

All three velocity-sensitive DINs have their soma, their main input dendrites, and some output branches in the gnathal ganglion (GNG, formerly known as subesophageal ganglion; Ito et al., 2014), and send a descending axon to the prothoracic ganglion (Fig. 1D). The somata of all three DINs are located in the ventral half of the GNG. Input branches were identified as smooth neurites (i.e., lacking blebs), whereas presynaptic sites were identified by their bleb-like structures (e.g., Heinze and Homberg, 2007). Morphological and functional descriptions are given with respect to the descending axon.

The contralateral ON-type velocity-sensitive DIN (cONv) was sensitive to movement of the contralateral antenna. cONv has its soma and putative input branches in the contralateral half of the GNG (Figs. 1Aii and 2Bi), and delivers output to both hemiganglia of the prothoracic ganglion (Fig. 1Aiii). From the prothoracic ganglion, $\mathrm{cONv}$ descends further toward downstream ganglia (descending axon in Fig. 1Aiii). cONv spikes could still be recorded in the connective entering the metathoracic ganglion. Hence, cONv potentially innervates motor networks of all three leg pairs. Additionally, cONv sends an ascending axon with bleblike arborizations through the ipsilateral GNG hemiganglion to the ipsilateral dorsal lobe of the deutocerebrum (Fig. 1Ai,Aiv; for localization, see Fig. $1 D$ ). Thus, putative $\mathrm{CONv}$ output branches extend to the ipsilateral hemiganglion of the GNG and to the brain. When stained in the same animal, the right and left cONv neurons form a bilaterally symmetrical, circular branching pattern around the center of the prothoracic ganglion (Fig. 1Aiii). The anatomy of cONv suggests that it receives input from the 

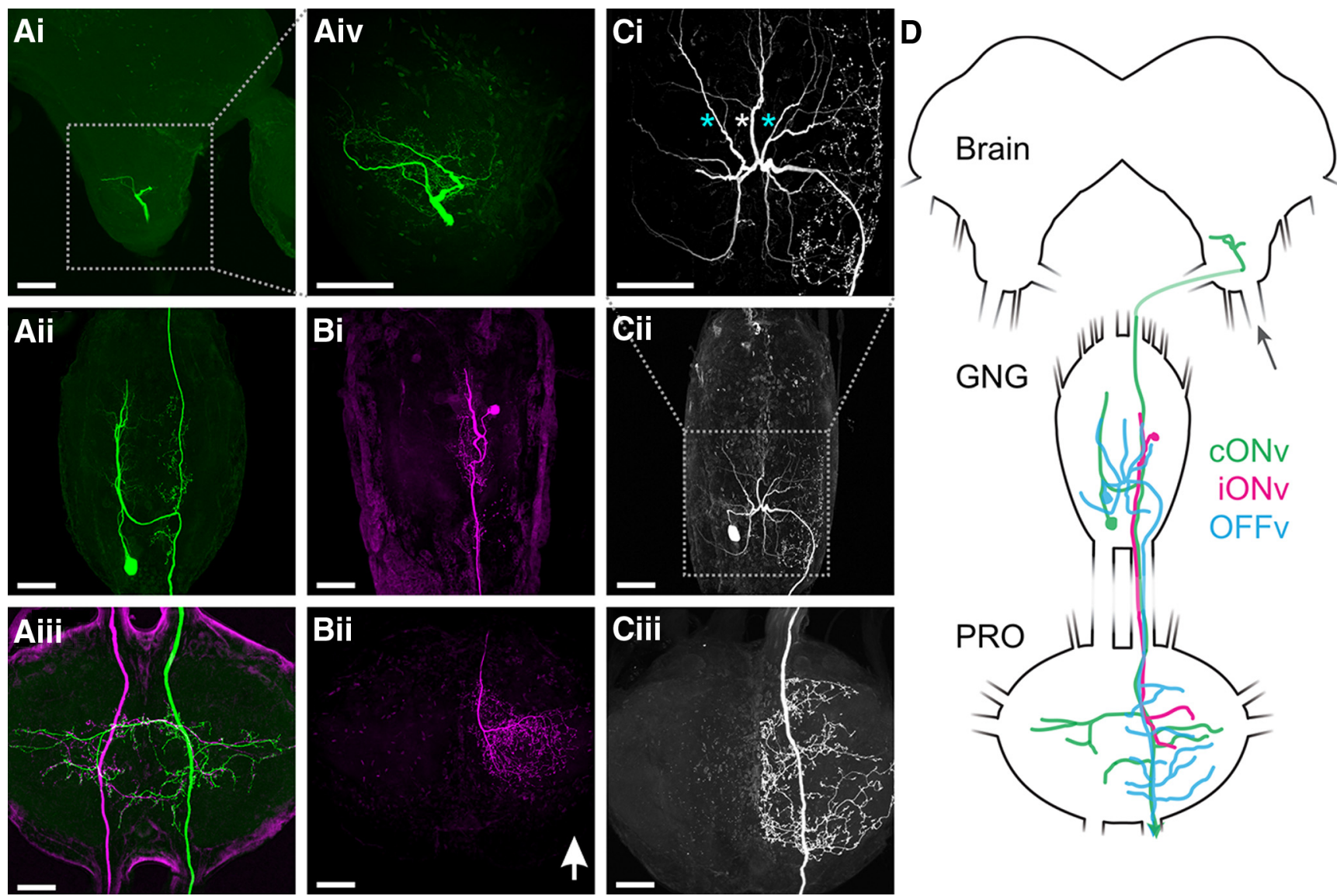

Figure 1. Morphology of identified velocity-sensitive DINs. $\boldsymbol{A}, \mathrm{CONv}$ (green) in the ipsilateral deutocerebrum of the brain (Ai)), the GNG (Aii)), and the prothoracic ganglion (Aiii). The contralateral cONv was stained in the same animal (magenta). The cONvs of both sides appear perfectly symmetrical. The green (magenta) cONv responded to movement of the left (right) antenna. Aiv, cONv branches in the ipsilateral deutocerebrum of the brain (indicated in $\boldsymbol{A i}$ ). The posterior part of the axon is not included in the stack. Bi, iONv branching pattern in the GNG. The right iONv responded to movement of the right antenna only. Bii, iONv branching pattern in the prothoracic ganglion. Ci, Cii, OFFv branching pattern in the GNG (location of Ciindicated in Cii). OFFv has three smooth main dendrites that run anteriorly in the GNG (asterisks) and bleb-like, putative presynaptic sites in the ipsilateral half of the GNG. OFFv responded to stimulation of both antennae. (iiii, 0 FFv branching pattern in the prothoracic ganglion. Scale bars, $100 \mu \mathrm{m}$. Arrow points anteriorly along the neuraxis. D, Schematic of the brain, GNG, and the prothoracic ganglion with drawings of cONv (green), iONv (magenta), and OFFv (cyan). Arrow indicates the antennal nerve. Connectives and axons are shortened.

contralateral antenna and forwards it to the ipsilateral half of the GNG, to the ipsilateral dorsal lobe of the brain, and to both halves of the prothoracic ganglion as well as to downstream thoracic ganglia (Fig. 3A).

In contrast, the ipsilateral ON-type velocity-sensitive DIN (iONv) was sensitive to movement of the ipsilateral antenna. iONv has its soma and mixed input and output branches in the ipsilateral half of the GNG (Figs. $1 \mathrm{Bi}$ and $2 \mathrm{Ci}$ ) and terminates in the prothoracic ganglion, with a spatially confined ipsilateral arborization (Fig. 1Bii). Hence, iONv innervates only the thoracic hemiganglion that controls movement of the ipsilateral front leg (Fig. 3B). The soma and descending axon of $\mathrm{iONv}$ are smaller than those of the other two velocity-sensitive DINs.

The OFF-type velocity-sensitive DIN (OFFv) was sensitive to movement of both antennae. OFFv sends three smooth main dendrites anteriorly in the GNG (Fig. 1Ci,ii, asterisks). The outer two of these branches (Fig. $1 C i$, cyan asterisks) run in a mediolateral direction rather than in the same tracts as the major $\mathrm{cONv}$ and $\mathrm{iONv}$ branches. Bleb-like, putative output branches in the GNG arise from more posterior branches and are restricted to the ipsilateral hemiganglion (Fig. 1Ci). Likewise, OFFv innervates only the ipsilateral half of the prothoracic ganglion (Fig. 1Ciii). The OFFv soma lies contralateral to the descending axon (Fig. 1Cii). OFFv descends further toward downstream ganglia and thus innervates at least the mesothoracic ganglion, which controls movements of the middle legs, as well (Fig. 1Ciii, descending axon). Therefore, the OFFv branching pattern suggests that it receives bilateral input in the GNG and forwards it to the ipsilateral halves of the GNG and the prothoracic ganglion, as well as to downstream thoracic ganglia (Fig. 3C).

Because all three DINs were sensitive to imposed movement of the SP joint, we tested whether DINs and antennal afferents might be directly connected in the GNG. To this end, we labeled axonal projections of proprioceptive hair fields that monitor SP joint movement (Okada and Toh, 2001), and subsequently stained DINs in the same preparations (Fig. 2). Antennal hair field afferents project to the dorsal lobe of the brain but also directly to the GNG (Fig. 2; see also Goldammer, 2013).

The putative output branches of $\mathrm{CONv}$ in the brain and hair field afferents from the SP joint project to the same region in the dorsal lobe (Fig. 2Ai). However, there is little, if no, direct overlap between $\mathrm{CONv}$ and hair field afferents on a finer scale, as can be seen in $10-\mu \mathrm{m}$-thick projections of the areas in which they come closest together (Fig. 2Aii,Aiii). Thus, $\mathrm{cONv}$ and hair field afferents do not seem to be directly connected in the dorsal lobe. They may, nonetheless, have the same postsynaptic targets (Fig. $3 A$ ).

In contrast, there is strong overlap between hair field afferents and DINs in the GNG (Fig. 2B,C). Projections of hair field affer- 
ents run in the same tracts as the main branches of cONv and $\mathrm{iONv}$ in the GNG (Fig. 2Bi,Ci). The smooth branches overlap strongly with hair field afferents in 10$\mu \mathrm{m}$-thick projections in several locations (an example is shown in Fig. 2Bii), whereas bleb-like branches and hair field afferents are mostly localized at slightly different depths (Fig. 2Biii) and only come into closer contact in one location. In keeping with the notion that smooth dendritic branches are input regions and bleb-like branches are output regions, these results suggest that the smooth contralateral cONv branches could be a direct postsynaptic target of antennal hair field afferents (Fig. 3A).

In case of $\mathrm{iONv}$, there is also considerable overlap between hair field afferents and the mixed iONv branches in the GNG. iONv runs in the same tract as the hair field afferents on a coarse scale (Fig. $2 \mathrm{Ci}$ ), and seems to overlap directly with hair field afferents on a fine scale (Fig. 2Cii). iONv may thus also receive direct input from antennal hair field afferents in the GNG (Fig. 3B).

To date, we did not obtain high-quality double labelings of OFFv and hair field afferents. However, OFFv has the most extensive branching pattern in the GNG, and some of its neurites may cross projection areas of hair field afferents (Fig. 1Ci, blue asterisks). Although it is thus possible that OFFv may receive direct input from antennal hair field afferents, it is very likely that the majority of connections between hair field afferents and OFFv are indirect, via interneurons. This is because mechanoreceptive afferent synapses are typically cholinergic and thus depolarizing (Stengl et al., 1990), whereas OFFv showed a decrease in firing rate upon antennal stimulation (see Fig. 8C). Direct synapses of hair field afferents produce the opposite effect of that observed in OFFv recordings.

The double-labeling experiments suggested that DINs receive input from antennal proprioceptors in the GNG (Fig. 2D). Hence, velocity-sensitive DINs form a direct pathway between antennal mechanoreceptors and thoracic networks, without receiving input in the brain (Fig. 3). Because all DINs originated from the GNG, and likely formed input as well as output synapses within the GNG, the GNG appears to be a key center for the integration of antennal mechanosensory information. This is in line with the presumed role of the GNG in the descending control of adaptive locomotion in insects, as was suggested by early behavioral experiments of Roeder (1937).

\section{ON-type velocity-sensitive DINs are highly sensitive to} antennal movement across the entire joint angle and velocity working range

Both iONv and cONv had a low baseline firing rate and responded strongly to imposed antennal movements (Fig. 4A,B). iONv responded to stimulation of the ipsilateral antenna (Fig.
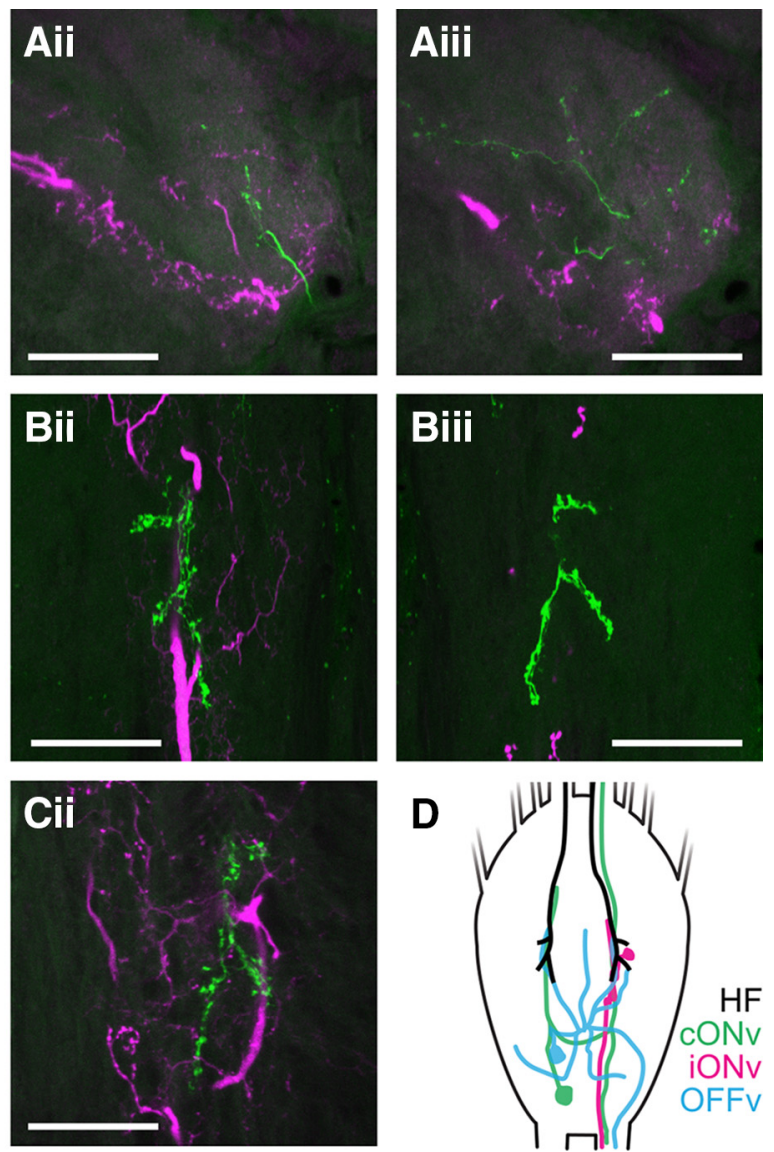

Figure 2. DIN neurites overlap with projections from antennal hair field afferents in the GNG. All double labelings of antennal hair field afferents (HF, green) and DINs (magenta) were obtained in the same individuals. Scale bars, $50 \mu \mathrm{m}$. Ai, Projections of 作 the main arborizations of $\mathrm{CONv}$, iONv, and OFFv in the GNG.

$4 A)$, whereas cONv responded to stimulation of the contralateral antenna (Fig. 4B). The view of direct afferent input to the DINs in the GNG is supported by physiological data, too: both cONv and iONv forwarded spikes to the thoracic ganglion rapidly. The response latency after antennal deflection was $11.2 \pm 2.7 \mathrm{~ms}$ in cONv (mean, $\mathrm{SD}, N=5, n=35)$, and $16.5 \pm 5.4 \mathrm{~ms}(N=5, n=$ $35)$ in $\mathrm{iONv}$, as measured in the prothoracic ganglion. This difference was statistically significant $(t=7.78, \mathrm{df}=34, p=3.3 \times$ $\left.10^{-5}\right)$. cONv thus forwarded spikes to the thorax slightly faster than iONv. However, both delays were short compared with the 40 ms required for the initiation of tactually induced leg movements (Schütz and Dürr, 2011), suggesting that velocity-sensitive DINs could contribute to tactually induced leg motor responses.

Other physiological properties of $\mathrm{cONv}$ and $\mathrm{iONv}$ were also similar, including their low resting activity (Fig. $4 A, B$ ). The resting activity of iONv was very low, at $0.60 \pm 0.42 \mathrm{~Hz}$ (mean $\pm \mathrm{SD}, N=$ 5) whenever the SP joint was held in the resting position. The resting activity of cONv was slightly higher than that of $\mathrm{iONv}$, but still low at $1.53 \pm 1.09 \mathrm{~Hz}(N=8)$. In both neurons, the spike frequency depended linearly on the joint angle velocity over a wide velocity range (Fig. $4 D$, E; correlation coefficients $\mathrm{R} \geq 0.98$ for all linear fits). Yet, the slope of the velocity-response charac- 

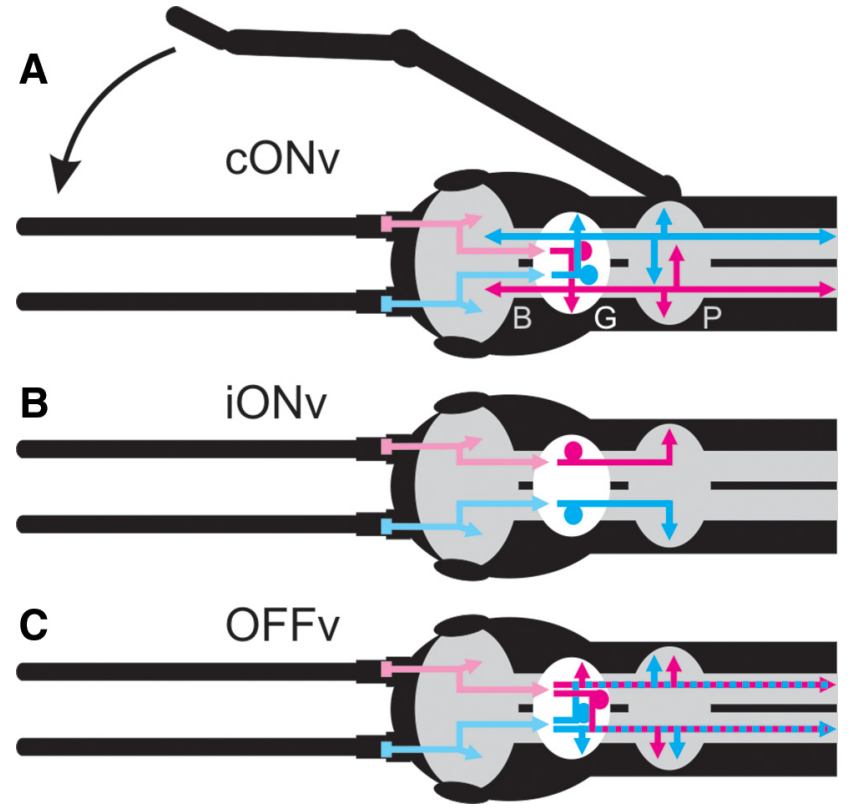

Figure 3. Parallel DINs with complementary properties convey the velocity of antennal movement to the prothoracic ganglion. Schematics of the identified velocity-sensitive DINs in the stick insect CNS, with afferent pathways from both antennae to the GNG (G). Magenta represents information from the right antenna. Cyan represents information from the left antenna. $A, C O N v$ receives contralateral input in the GNG and mediates it to both sides of the prothoracic ganglion (P), which controls front leg movement. CONv also innervates the brain (B) and ganglia downstream from the prothoracic. Black arrow indicates the behavioral results: stick insects use antennal cues to guide reaching movements. $\boldsymbol{B}$, iONv receives ipsilateral input in the GNG and mediates it to the ipsilateral half of the prothoracic ganglion. C, OFFv receives bilateral input in the GNG and mediates it to the ipsilateral half of the prothoracic ganglion. All DINs have their soma (filled circles) and antennal mechanosensory input regions in the GNG. Arrows indicate output branches in the respective hemiganglia.

teristic of $\mathrm{iONv}$ was twice as steep as that of $\mathrm{cONv}$ (Fig. $4 F$ ) because the spike rate of $\mathrm{iONv}$ was about twice that of $\mathrm{cONv}$ at any given velocity. This difference was significant $(t=5.83, \mathrm{df}=$ $10, p=1.65 \times 10^{-4}$; Fig. $\left.4 F\right)$. Therefore, both iONv and cONv linearly encoded the SP joint angle velocity over a wide velocity range, but iONv had twice the gain of cONv. This difference in gain between $\mathrm{iONv}$ and $\mathrm{cONv}$ might reflect the relative importance of the right and left antenna for the right and left front legs, respectively. For example, only the ipsilateral front leg has been shown to execute a retargeting movement in response to an antennal contact event, and bilateral reaching movements are less common than ipsilateral ones (Schütz and Dürr, 2011).

Large-amplitude movements of the SP joint often elicited spike trains with fluctuating spike frequency, especially at relatively low joint angle velocities (Fig. $4 A, B$ ). These fluctuations could be due to low reliability, an additional position-sensitive component in the response, or an effect of sustained, constant stimulation. To test this, we applied a sequence of smallamplitude movements within the entire SP joint working-range (Fig. 4C). This type of stimulus elicited sequences of reliable and strong responses in ON-type DINs, across the entire working range of the SP joint (Fig. $4 C$ ). These responses were largely independent of the joint angle and also occurred in joint angle ranges in which few or no spikes were elicited during largeamplitude ramps (compare Fig. $4 A, C$ ). Therefore, spike frequency fluctuations during large-amplitude movements were primarily a consequence of sustained stimulation (e.g., due to short-term adaptation). Hence, $\mathrm{iONv}$ and $\mathrm{cONv}$ consistently respond to antennal movement within the entire working range of the SP joint. Other stimulus parameters, such as joint angle or movement direction, modulated the response of velocity-sensitive DINs. These effects, however, were much smaller than the effects of changes in joint angle velocity. A purely data-driven analysis of the coding properties of these DINs reveals that motion sensitivity is indeed one of two main descriptors (the other being position sensitivity). According to this analysis, both ONv DINs and OFFv exhibit a weak position selectivity, along with very pronounced velocity sensitivity (J.M.A. and V.D., submitted).

The linear response characteristics, the wide velocity sensitivity range covering three orders of magnitude, and the high peak firing rates of up to $300 \mathrm{~Hz}$ indicated that the input driving both $\mathrm{cONv}$ and $\mathrm{iONv}$ was generated by velocity-sensitive proprioceptors at the SP joint. Given the morphological overlap between the terminals of antennal hair field afferents and dendrites of $\mathrm{ON}$ type velocity-sensitive DINs, the response is likely driven by afferents of the SP joint hair fields. This is also supported by a DIN model, which can explain the activity of ON- and OFF-type velocity-sensitive DINs by assuming they only receive input from hair field afferents (J.M.A. and V.D., submitted).

In addition to the antennal movement response, $\mathrm{cONv}$ was sensitive to gentle taps onto the recording table, which elicited substrate vibrations (Fig. $5 A$, left). This vibration response prevailed after ablation of both antennae, and after transection of the neck-connectives posterior to the recording site. Hence, the vibration response must have been driven by mechanoreceptors on the head, neck, or mouth parts. Thus far, the identity of these receptors is not clear, but they are certainly not part of the antennal system.

Systematic analysis of this vibration response showed that almost all substrate contacts elicited a single spike in $\mathrm{cONv}$ (Fig. $5 C$ ). As a consequence, the firing frequency of $\mathrm{cONv}$ could be controlled by the tapping frequency (Fig. 5B). Moreover, whenever extracellular whole-nerve recordings from the neckconnectives were obtained in addition to an intracellular cONv recording, cONv was identified as the largest unit in the wholenerve recordings (Fig. $5 \mathrm{C}$ ). Therefore, it was possible to conduct double recordings from both $\mathrm{CONv}$ neurons or from $\mathrm{CONv}$ and a different DIN, combining one intracellular recording with the extracellular hook-electrode recording of cONv. Exploiting its unique response to substrate vibration and its large extracellular spike amplitude allowed unmistakable identification and reliable, long-lasting recordings of $\mathrm{cONv}$ in virtually any specimen, and in various setups. As a result, cONv offers a unique opportunity for studying neural network properties involving cONv, but also simultaneous, complementary coding of antennal movement in multiple DINs. For example, it was possible to record from both the right and the left cONv in parallel within the same animal, showing that each cONv only responded to movement of the contralateral antenna (Fig. 6A, $B$ ).

Whereas staircase stimuli were ideal to characterize DIN selectivities to antennal movement, position, and direction, natural antennal movements are better described by sinusoids (Krause and Dürr, 2004; Krause et al., 2013). When using sinusoidal stimuli, the $\mathrm{CONv}$ spike frequency was always proportional to the joint angle velocity (Fig. 6C). This velocity sensitivity persisted even at SP joint cycle frequencies as low as $0.005 \mathrm{~Hz}$, with a cycle period longer than $3 \mathrm{~min}$ and peak velocities of $<1.6 \%$ (Fig. $6 D$ ). Therefore, cONv was sensitive to SP joint movement across the entire, naturally occurring amplitude and velocity range, from $0.4^{\circ} \mathrm{s}$ up to at least $800^{\circ} \mathrm{s}$.

Because of the extracellular recordability, we could also directly test whether the ascending cONv collateral in the brain was 
A

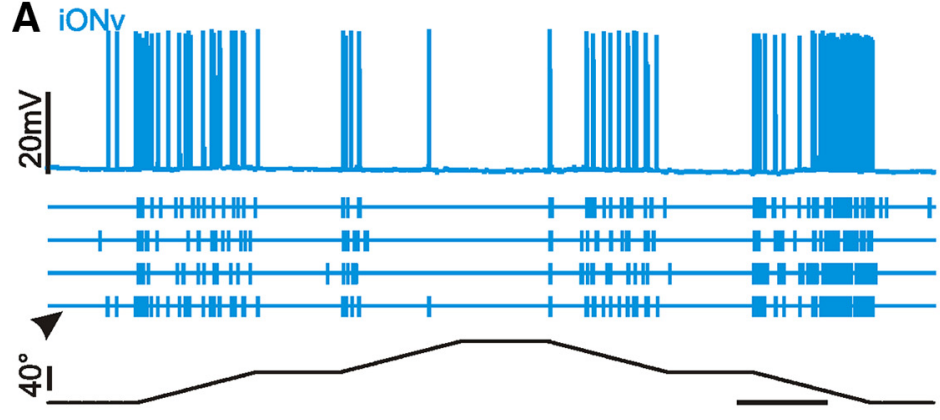

B cONv

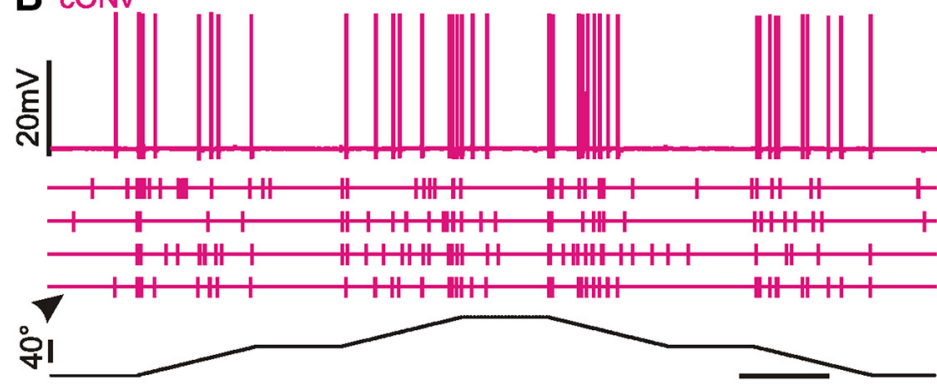

C iOnv

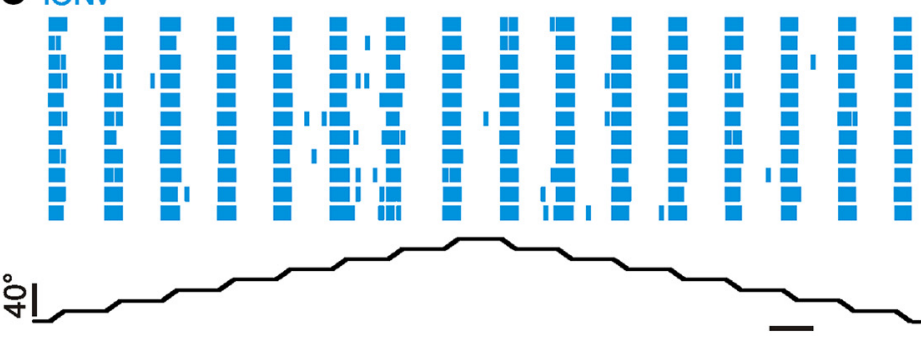

D
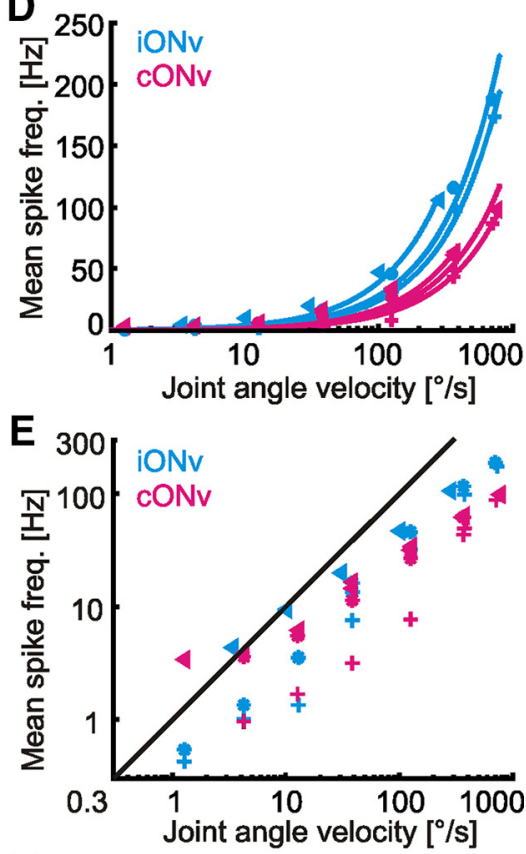

F 0.5

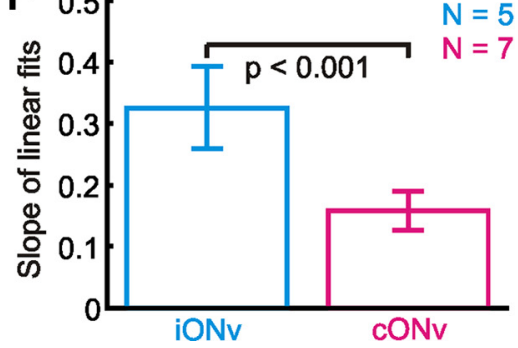

Figure 4. iONv and CONv are highly sensitive to antennal movement and encode the antennal joint angle velocity over a wide velocity range. $\boldsymbol{A}$, Response of iONv (5 top traces, cyan) to SP joint stimulation (bottom trace, black). Arrowhead indicates the sweep shown as the membrane potential (top). $\boldsymbol{B}$, cONv response to the same stimulus as for i0Nv in $\boldsymbol{A}$. Details as in $\boldsymbol{A}$. $\boldsymbol{C}$, Vertical lines (cyan) indicate iONv spikes during 11 consecutive sweeps of SP joint stimulation with 16 low-amplitude ramps (bottom). $D$, Mean spike frequencies of iONv und c0Nv during stimulation at different velocities in three different animals each (different symbols), shown on a log/lin scale. Frequencies were averaged across all four ramps of each staircase stimulus. The lines show linear fits ( $R \geq 0.98$ for all fits). iONv had a higher mean spike rate than cONv for a given velocity. $\boldsymbol{E}$, Velocity response characteristics of iONv (cyan) and cONv shown on a log/log scale. Different symbols represent data from three different animals. Black line indicates spike frequency (in $\mathrm{Hz}$ ) = joint angle velocity (in \%), for reference. The spike frequency was proportional to the velocity even at slow joint angle velocities between $1 \% \mathrm{~s}$ and $40 \%$ s. $\boldsymbol{F}$, Slopes of iONv and cONv velocity sensitivity ( $\boldsymbol{D}$, mean $\pm \mathrm{SD})$. $\boldsymbol{A}-\boldsymbol{C}$, Horizontal scale bars indicate $1 \mathrm{~s}$; ramp velocities were $40 \% \mathrm{~s}$.

an input or an output branch. For this, we recorded the left and right $\mathrm{cONv}$ in one animal and transected one circumoesophageal connective between the GNG and the brain while leaving the other intact (Fig. 7A). The transection ablated the ascending neurite of only one cONv, and it additionally silenced the descending afferent terminals on that side. We reasoned that, depending on which one of the two cONvs reduced firing during antennal movement after the transection, we could tell whether afferent input was delivered to the dorsal lobe of the brain or to the GNG (Fig. 7A). Before transection, the left $\mathrm{cONv}$ responded to movement of the right antenna (Fig. $7 \mathrm{Bi}$ ), whereas the right $\mathrm{cONv}$ responded to movement of the left antenna, as expected (Fig. $7 \mathrm{Bii}$ ). After transection of the right circumoesophageal connective, the response of the right $\mathrm{cONv}$, whose ascending branch was cut, remained unaffected (Fig. 7Cii). In contrast, the response of the left cONv was greatly diminished (Fig. 7Ci), despite the fact that its ascending branch was still intact. This indicated that the transection of the descending afferent fibers in the circumoesophageal connective must have caused the decline of the response. This proves that $\mathrm{CONv}$ receives input in the GNG, and not in the brain. We conclude that the ascending branch of $\mathrm{cONv}$ and its arborization in the brain do not receive the sensory input that is driving the antennal movement response of cONv. Indeed, parallel extracellular recordings from several positions along the ventral nerve cord revealed that $\mathrm{CONv}$ spikes were ascending from the GNG to the brain and descending from the GNG to the metathoracic ganglion (data not shown).

The very weak response of the left, intact $\mathrm{cONv}$ that persisted after transection of the circumoesophageal connectives (Fig. 7Ci) suggests that transection did not completely abolish the stimulus-induced response, despite the fact that the largest fraction of the response had vanished. This small remainder of the stimulus-induced activity may either have been mediated through the vibration-sensitive input or via interneurons mediating antennal movement information across the midline of the brain.

The vibration response remained unaffected in both the right and the left cONv after transection (Fig. 7D). Therefore, the vibration response is driven by nondescending mechanoreceptors projecting directly to the GNG. All of this suggests that the ascending cONv collateral conveys a copy of the descending antennal tactile information from the GNG to the contralateral brain hemisphere, at least during antennal and vibration stimuli. 
A

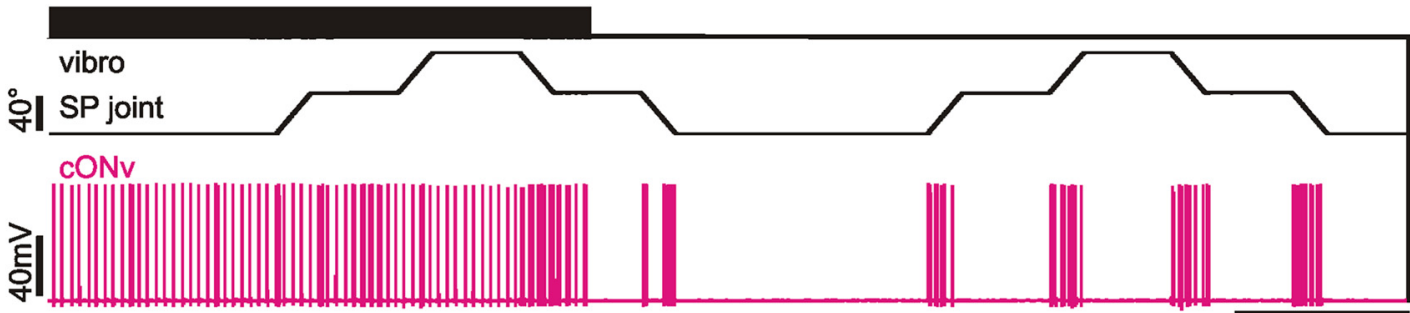

B Inst. frequency.
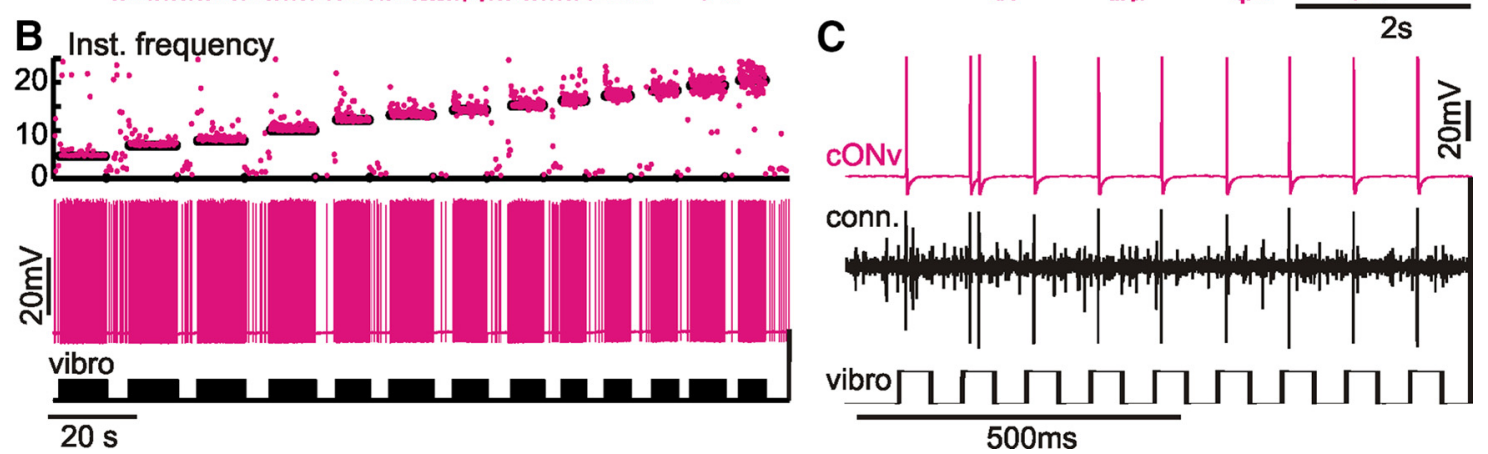

Figure 5. CONv is sensitive to substrate vibration and is the largest unit in extracellular neck-connective recordings. $\boldsymbol{A}$, The sequence shows the c0Nv response to substrate taps (vibro) alone (left), taps with simultaneous SP joint stimulation (first staircase), and SP joint stimulation only (second staircase). $\boldsymbol{B}$, Intracellular cONv recording during different substrate tapping frequencies. The instantaneous spike frequency plot (top) shows that cONv (magenta) closely followed the substrate tapping frequency (black). $C$, Intracellular c0Nv recording and whole-nerve neck-connective recording (conn.) during substrate tapping. Vibrator contact was induced on the rising edge of the stimulus monitor (bottom). coNv was the largest unit in whole-nerve recordings.

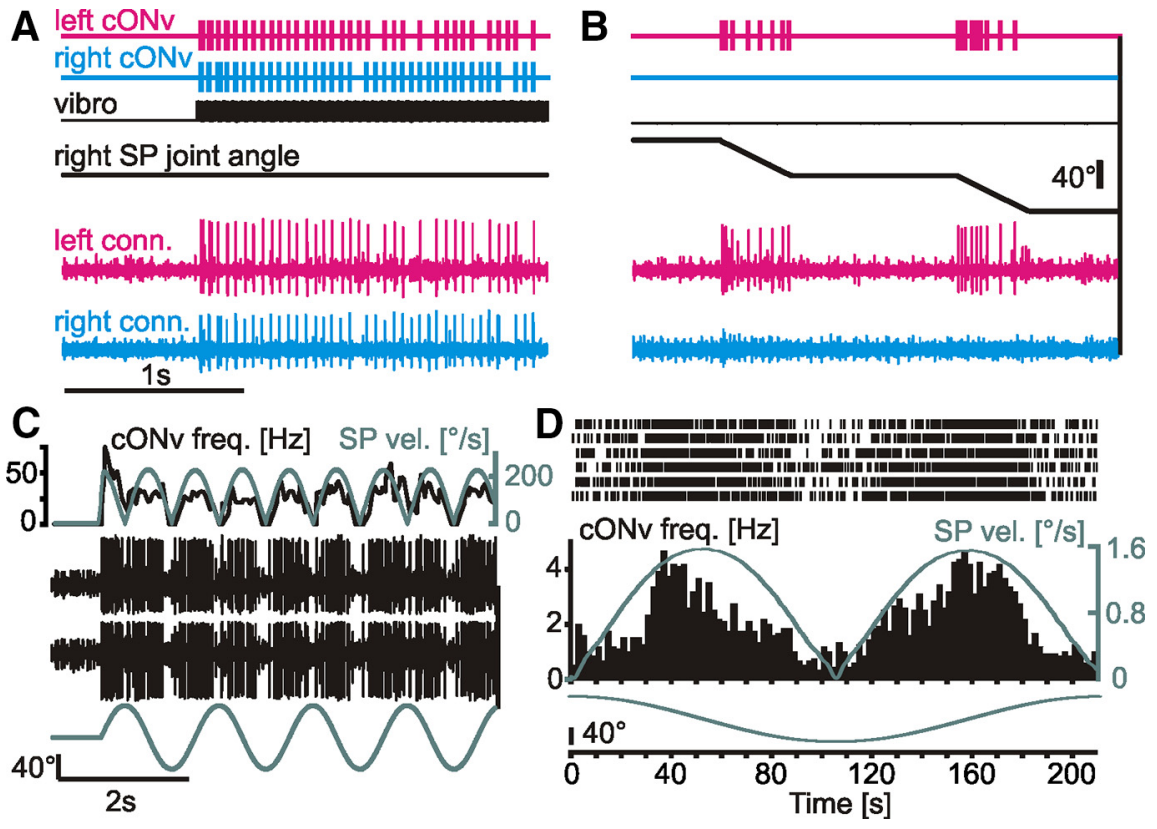

Figure 6. cONv exclusively receives contralateral antennal input and is sensitive to sinusoidal movement of the SP joint, even at very slow velocities. $A, B$, Bottom, Simultaneous recordings of the left (magenta) and right (cyan) neck-connectives. CONv was the largest unit in both recordings. Top two traces represent the timing of CONv spikes, extracted by thresholding the extracellular recordings of the neck connectives. Middle two traces represent the tapping stimulus (vibro) and the right SP joint angle. The left and right $C O N v$ responded to the tapping stimulus $(\boldsymbol{A})$, but only the left $C O N v$ responded to stimulation of the right $S P$ joint (B). C, CONv response to sinusoidal movement of the SP joint at $0.69 \mathrm{~Hz}$ (bottom), with simultaneous neck-connective recordings at two different positions on the same side (middle black traces). cONv was the largest unit in both recordings. The spike frequency of $\mathrm{CONv}$ (top black line) followed the time course of the SP joint angle velocity (top gray line). D, Same type of experiment as in $C$, but with very low stimulus velocity. CONv spiked reliably (raster plot with PSTH below) during 6 consecutive cycles of sinusoidal SP joint movement at $0.0047 \mathrm{~Hz}$. Bottom gray trace represents SP joint angle. Middle gray trace represents stimulus velocity. The spike frequency of CONv follows the time course of SP joint angle velocity at velocities exceeding $0.4^{\circ} \%$. C, D, Velocity traces were averaged, using a $50 \mathrm{~ms}$ time window. $\boldsymbol{C}$, The mean spike frequency was averaged, using a $100 \mathrm{~ms}$ time window.
Combined processing of antennal movement by $\mathrm{ON}$ - and OFF-type DINs

The response characteristics of $\mathrm{cONv}$ and $\mathrm{iONv}$ on the one hand, and of OFFv on the other, were similar but inverted. This suggested that ON- and OFF-type velocity-sensitive DINs might exert a combined effect on downstream neurons in thoracic motor control networks, provided they deliver synchronous output to the prothoracic ganglion. To test this, $\mathrm{cONv}$ and $\mathrm{OFFv}$ were recorded in parallel. First, cONv was recorded intracellularly and extracellularly (Fig. 8A). Averages of cONv spikes did not differ in shape when triggered by intracellular or extracellular spikes (Fig. 8B), and both recordings detected almost identical numbers of spikes. This confirmed that $\mathrm{CONv}$ spikes were reliably detected in the extracellular recording. Next, the intracellular recording of $\mathrm{CONv}$ was terminated and the ipsilateral OFFv was recorded intracellularly instead, whereas the extracellular cONv recording was maintained (Fig. 8C). Whenever the right antenna moved, the spike frequency of OFFv decreased while cONv started to spike (Fig. $8 C, E$ ). The faster the antenna moved, the lower was the spike rate in OFFv. This typical velocity response characteristic of OFFv is shown in Figure $8 D$. Cross-correlation of the spike trains of $\mathrm{CONv}$ and $\mathrm{OFFv}$ revealed a negative peak with nearly no time lag (Fig. $8 F)$. This shows that $\mathrm{CONv}$ and OFFv de- 
livered synchronous output to the thoracic ganglia, albeit with opposite sign. Given the small difference in delays between $\mathrm{iONv}$ and cONv, we assume that both ON-type DINs also delivered synchronous output to the prothoracic ganglion.

In summary, $\mathrm{cONv}, \mathrm{iONv}$, and $\mathrm{OFFv}$ act together in mediating information about tactile sensor movement to the prothoracic ganglion at any given moment. As a triplet, they mediate fast information about antennal movement to thoracic motor networks and are thus well suited to drive adaptive motor responses, such as fast, tactually induced front leg reaching. Primarily, these DINs seem to be involved in the spatial coordination of antenna and leg movements during locomotion, which has to occur during every step an animal takes. Although the overall behavioral patterns, walking or climbing, are neither strikingly fast nor highly specialized, they nonetheless require the fast and precise transmission of information from tactile sensors to thoracic motor networks.

\section{Discussion}

Descending pathways are at the intersection between sensory and motor networks and therefore serve an important role in determining behavioral output. Here, we characterized a novel descending pathway that mediates proprioceptive information from tactile sensors on the head to thoracic neural networks. The neurons of this pathway have different but complementary response patterns and morphologies. We analyzed how they act together to encode tactile sensor movement.

\section{Velocity-sensitive DINs mediate detailed information about antennal movement directly to thoracic neural networks}

In insects, leg movements are controlled by thoracic interneurons, which are rhythmically active during locomotion and integrate sensory information from mechanoreceptors to drive motoneurons (Burrows and Siegler, 1976; Büschges et al., 1994; Burrows, 1996). Many of these interneurons are restricted to the thoracic hemiganglion ipsilateral to the leg they control (Siegler and Burrows, 1979; Büschges, 1990). Hence, DIN branching patterns in the thoracic ganglia likely indicate whether they act upon motor networks of the right or left side of the body (e.g., Gonzalez-Bellido et al., 2013).

The selectivities of the velocity-sensitive DINs for movement of the right or left antenna and their thoracic branching patterns suggest that $\mathrm{iONv}$ mediates information about the movement of the ipsilateral antenna to the ipsilateral front leg, cONv mediates information about the movement of the contralateral antenna to both front legs and downstream ganglia, and OFFv mediates information about the movement of both antennae to the ipsilateral front leg and downstream ganglia. Because copies of the same three DINs exist on both sides of the CNS (e.g., see
Fig. 1Aiii), this small set of DINs provides both halves of the prothoracic ganglion with information about the movement of both antennae (Fig. 3). This information is unambiguous with respect to which antenna moved at what velocity.

The opposite signs of the ONv and OFFv responses, and the fact that they occur synchronously, appear ideal for a push-pull mechanism, like that found in the fly visual system (Borst and Euler, 2011). Assuming that downstream neurons are inhibited by OFFv and excited by iONv and cONv, a push-pull mechanism could be one way to precisely modulate thoracic premotor neurons.

This is rather different from other descending mechanosensory systems. Touch-sensitive DINs in cockroaches and crickets, for example, all respond to antennal contact events or wind stimuli with relatively few spikes (Burdohan and Comer, 1996; Schöneich et al., 2011). Therefore, they constitute parallel pathways with similar response properties. Several descending brain interneurons, which encode parameters of antennal posture and movement, were identified in the cricket (Gebhardt and Honegger, 2001). There, different DINs cover different parts of the joint 


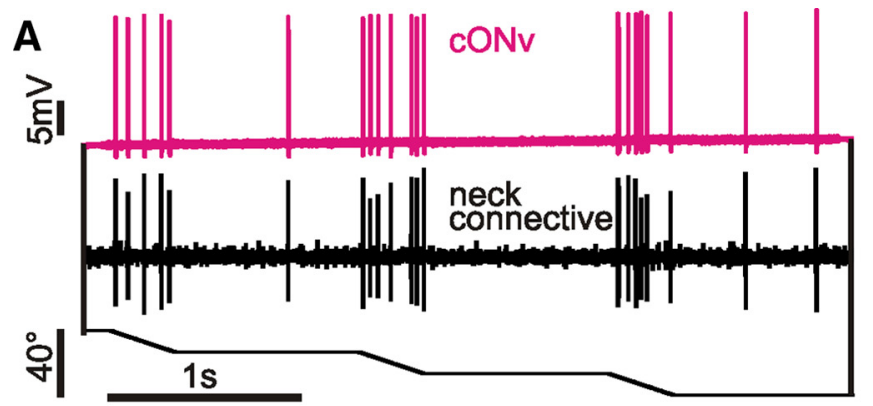

B
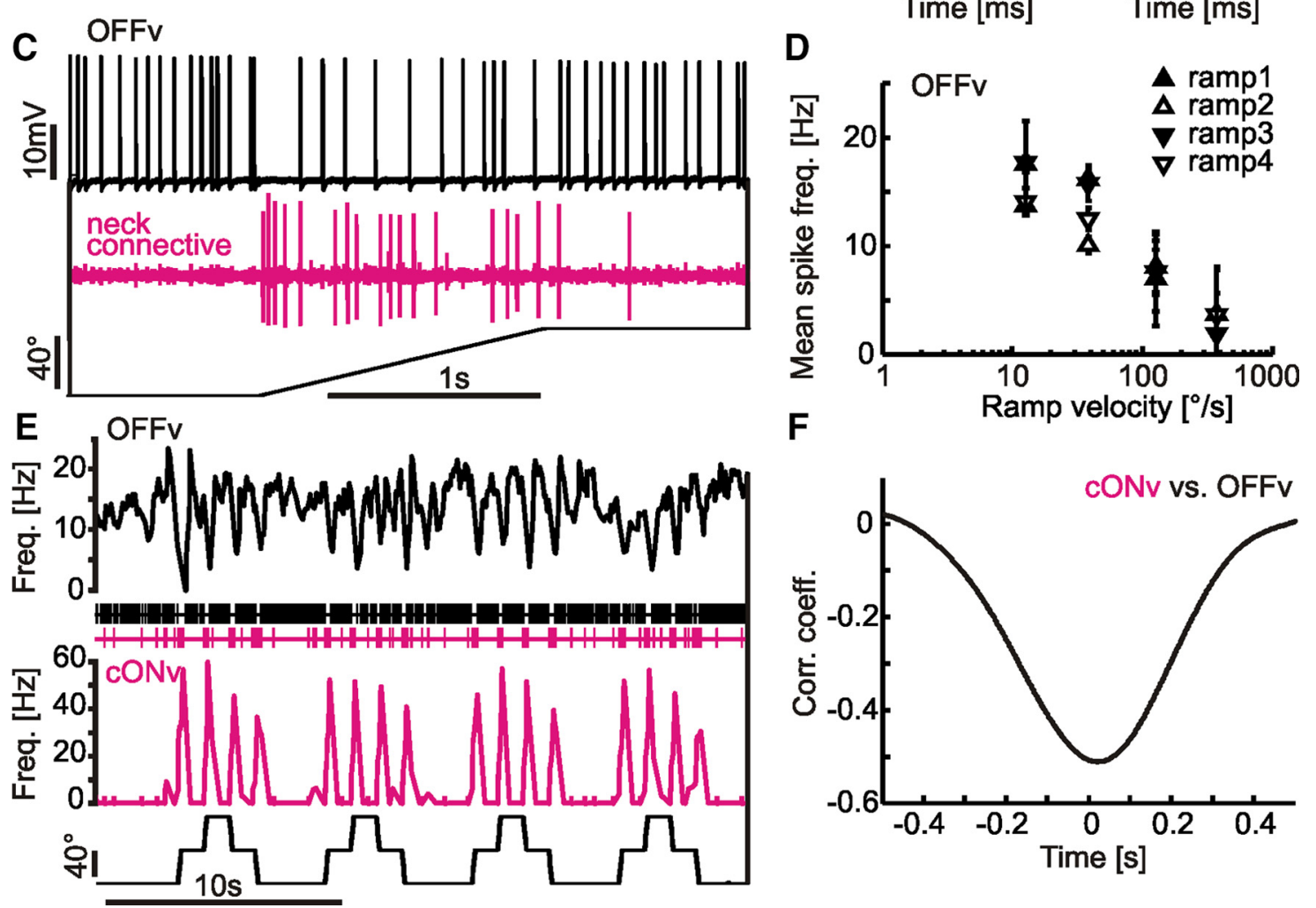

Figure 8. CONv and OFFv deliver synchronous output to prothoracic networks. All data are from one continuous experiment. $A$, Intracellular cONv and neck-connective recordings during SP joint movement (bottom). $\boldsymbol{B}$, Spike-triggered average of the intracellular cONv recording using either the intracellular (magenta) or the extracellular cONv spike (black) as the trigger. Right, Both averages were overlaid, revealing identical time courses. cONv spikes can be extracted from the neck-connective recording with $>99 \%$ reliability. C, Intracellular OFFv recording (top), with the simultaneous neck-connective recording (middle, magenta) showing cONv spikes in response to antennal deflection (bottom). $\boldsymbol{D}$, Mean spike rate of OFFv during ramps with different velocities. $\boldsymbol{E}$, Mean spike rates of OFFv (black) and CONv (magenta) with the respective spike trains (vertical lines in the middle traces) and the stimulus (bottom). Spike rates were averaged within a 33 ms sliding window. $\boldsymbol{F}$, Cross-correlation of CONv and OFFv spike rates, as shown in $\boldsymbol{E}$, within a 145-s-long time window. Spike rates were normalized by subtracting the median rate.

angle working range, such that the firing pattern of an individual DIN provides mainly positional information. In contrast, all velocity-sensitive DINs described here respond to movement across the entire joint angle working range with persistent changes in their firing rate up to $250 \mathrm{~Hz}$. This indicates that a rate code is meaningful in this pathway rather than the timing of individual spikes. The sensitivity ranges of velocity-sensitive DINs overlap completely, in the angular as well as the velocity range covered by natural antennal movements. The three velocity-sensitive DINs therefore encode the same aspects of antennal movement, but using complementary response properties, inputs, and gains. Thus, velocity-sensitive DINs mediate graded information about tactile sensor movement to the thorax.

This movement-sensitive pathway connects the antennae directly to thoracic networks, bypassing higher processing and motor control centers in the brain, such as the central complex (Bender et al., 2010; Guo and Ritzmann, 2013; Seelig and Jayara- man, 2013). These higher brain centers may have a modifying influence on the descending signals, but they are no immediate component of this pathway. The tuning characteristics of velocity-sensitive DINs reflect those of rather simple, phasic mechanoreceptors (Matheson, 1992; e.g., Okada and Toh, 2001). Therefore, the velocity-sensitive DINs essentially forward raw data to thoracic neural networks, which must integrate and transform these simple signals to drive appropriate changes in leg movements for adaptive locomotion. The advantage of such a direct, short-latency pathway may be that leg movements can be directly influenced by antennal cues, permitting their fast adaptation. Locomotion, including complex patterns as occur during climbing, may therefore emerge in a kind of autopilot mode, leaving computational brain resources free for other tasks.

Büschges (1989) described ON- and OFF-type velocity-sensitive interneurons in the stick insect that responded to movement of a leg joint. These neurons originated from the mesothoracic ganglion 
but innervated other thoracic ganglia as well. One of them formed a ring-like structure around the center of the mesothoracic ganglion and had prominent lateral output branches. The morphology of the neuron was thus very similar to that of cONv (Büschges, 1989; his Fig. $3 A$ ). The similarities of the response properties and arborization patterns suggest that $\mathrm{CONv}, \mathrm{iONv}$, and OFFv could be part of an interneuronal coordination chain, which is spanning the antennae and all legs. Indeed, stick insects do not only target front legs toward antennal contact locations, but also other legs toward the touchdown location of their respective anterior leg (Theunissen et al., 2014). Therefore, velocity-sensitive DINs likely feed directly into thoracic neural networks coordinating movements of all limbs during adaptive locomotion.

\section{cONv, a new giant DIN}

Apart from their putative role in intersegmental coordination, velocity-sensitive DINs could also mediate touch-related cues. For example, antennal deflections, such as those used in our experiments, occur during contact with an approaching predator or with an object during locomotion.

Moreover, the vibration sensitivity of cONv might signal an approaching predator and could thus add long-range sensitivity to this near-range tactile pathway. Typically, insects sense substrate-borne vibrations with the subgenual organs in their legs (e.g., Hill, 2009) and airborne vibrations with Johnston's organ of the antenna (Staudacher et al., 2005). However, ablation of the antennae and transection of the neck-connectives revealed that the vibration sensitivity of $\mathrm{cONv}$ was driven by neither antennal nor leg mechanoreceptors. Although the input driving the vibration response remains elusive, the vibration sensitivity is nonetheless important for the identification of cONv across animals.

$\mathrm{cONv}$ has a large-diameter axon, is fast-mediating, and can be recorded extracellularly. These properties are shared with escapemediating giant visual DINs, such as the descending contralateral movement detector of the locust (O'Shea and Williams, 1974; Gabbiani et al., 1999) or the dipteran giant fiber system (Levine and Tracey, 1973; Tanouye and Wyman, 1980; Bacon and Strausfeld, 1986). Descending contralateral movement detector and the giant fiber provide important insights into the descending control of behavior because they can be recorded and identified across individuals, in a variety of setups (Fotowat et al., 2011; von Reyn et al., 2014). cONv adds to this collection of accessible, identified DINs and thus has the potential to fuel future research into active tactile sensing and descending motor control.

\section{Similarities of tactile and descending pathways between insects and mammals}

Three features of the descending pathway described here have counterparts in mammalian tactile sensory and descending motor pathways.

First, velocity-sensitive DINs of the stick insect mediate information about antennal movement via ON and OFF pathways. Similarly, whisker movement is encoded by ON- and OFF-type interneurons in mice, which might form a push-pull mechanism driving downstream neurons (Gentet et al., 2012; Petersen, 2014). ON- and OFF-type units were also recorded in the cockroach central complex during antennal stimulation (Ritzmann et al., 2008). Hence, ON and OFF pathways might be a general feature of tactile pathways in insects and mammals alike. Here, we demonstrate an ON/OFF pathway that directly mediates information from tactile sensors to thoracic motor networks.

Second, iONv mediates antennal cues exclusively to the ipsilateral half of the prothoracic ganglion. In contrast, $\mathrm{cONv}$ de- scends to the metathoracic ganglion and OFFv at least to the mesothoracic ganglion. In mice, more brainstem nuclei target motor neurons of front legs than of hind legs. This corresponds to the larger diversity and higher precision of front leg movements (Esposito et al., 2014). Similarly, the prothoracic ganglion controlling highly variable front leg movements in the stick insect is the only ganglion receiving input from all DINs.

Third, cONv forwards a copy of the descending signal from the GNG to the brain. Velocity-sensitive DINs seem to be part of a descending pathway that mediates the retargeting of front leg movements to an antennal contact position (Schütz and Dürr, 2011). This requires control of front leg movements through descending signals. Refinement of targeted limb movements relies on internal feedback loops (Scott, 2004). With its ascending branch, cONv may be part of a feedback loop that informs the brain about descending premotor signals. Similarly, vertebrate motor systems feature polysynaptic pathways that forward copies of descending motor signals back to the brain (Alstermark et al., 1981). These feedback loops are crucial for the control of aimed front leg movements in mice (Azim et al., 2014). cONv, with its ascending branch, may provide such feedback as a single neuron.

Together, the triplet of velocity-sensitive DINs is another remarkable example of how insects implement complex sensorimotor pathways with few neurons in small nervous systems.

\section{References}

Ache JM, Dürr V (2013) Encoding of near-range spatial information by descending interneurons in the stick insect antennal mechanosensory pathway. J Neurophysiol 110:2099-2112. CrossRef Medline

Alstermark B, Lindström S, Lundberg A, Sybirska E (1981) Integration in descending motor pathways controlling the forelimb in the cat: 8 . Ascending projection to the lateral reticular nucleus from $\mathrm{C} 3-\mathrm{C} 4$ propriospinal also projecting to forelimb motoneurones. Exp Brain Res 42:282-298. Medline

Azim E, Jiang J, Alstermark B, Jessell TM (2014) Skilled reaching relies on a V2a propriospinal internal copy circuit. Nature 508:357-363. CrossRef Medline

Bacon JP, Strausfeld NJ (1986) The dipteran giant fibre pathway: neurons and signals. J Comp Physiol A Neuroethol Sens Neural Behav Physiol 158:529-548. CrossRef

Bender JA, Pollack AJ, Ritzmann RE (2010) Neural activity in the central complex of the insect brain is linked to locomotor changes. Curr Biol 20:921-926. CrossRef Medline

Bidaye SS, Machacek C, Wu Y, Dickson BJ (2014) Neuronal control of Drosophila walking direction. Science 344:97-101. CrossRef Medline

Bläsing B, Cruse H (2004) Mechanisms of stick insect locomotion in a gapcrossing paradigm. J Comp Physiol A Neuroethol Sens Neural Behav Physiol 190:173-183. CrossRef Medline

Borst A, Euler T (2011) Seeing things in motion: models, circuits, and mechanisms. Neuron 71:974-994. CrossRef Medline

Burdohan JA, Comer CM (1996) Cellular organization of an antennal mechanosensory pathway in the cockroach, Periplaneta americana. J Neurosci 16:5830-5843. Medline

Burrows M (1996) The neurobiology of an insect brain. Oxford, United Kingdom: Oxford UP.

Burrows M, Siegler MV (1976) Transmission without spikes between locust interneurones and motoneurones. Nature 262:222-224. CrossRef Medline

Büschges A (1989) Processing of sensory input from the femoral chordotonal organ by spiking interneurones of stick insects. J Exp Biol 144: 81-111.

Büschges A (1990) Nonspiking pathways in a joint-control loop of the stick insect Carausius morosus. J Exp Biol 151:133-160.

Büschges A (2012) Lessons for circuit function from large insects: towards understanding the neural basis of motor flexibility. Curr Opin Neurobiol 22:602-608. CrossRef Medline

Büschges A, Kittmann R, Schmitz J (1994) Identified nonspiking interneurons in leg reflexes and during walking in the stick insect. J Comp Physiol A Neuroethol Sens Neural Behav Physiol 174:685-700.

Camhi JM, Johnson EN (1999) High-frequency steering maneuvers medi- 
ated by tactile cues: antennal wall-following in the cockroach. J Exp Biol 202:631-643. Medline

Comer CM, Parks L, Halvorsen MB, Breese-Terteling A (2003) The antennal system and cockroach evasive behavior: II. Stimulus identification and localization are separable antennal functions. J Comp Physiol A Neuroethol Sens Neural Behav Physiol 189:97-103. Medline

Diamond ME, von Heimendahl M, Knutsen PM, Kleinfeld D, Ahissar E (2008) 'Where' and 'what' in the whisker sensorimotor system. Nat Rev Neurosci 9:601-612. CrossRef Medline

Dirks JH, Dürr V (2011) Biomechanics of the stick insect antenna: damping properties and structural correlates of the cuticle. J Mech Behav Biomed 4:2031-2042. CrossRef Medline

Dürr V (2014) Stick insect antennae. Scholarpedia 9:6829. CrossRef

Dürr V, König Y, Kittmann R (2001) The antennal motor system of the stick insect Carausius morosus: anatomy and antennal movement pattern during walking. J Comp Physiol A Neuroethol Sens Neural Behav Physiol 187:131-144. CrossRef Medline

Esposito MS, Capelli P, Arber S (2014) Brainstem nucleus MdV mediates skilled forelimb motor tasks. Nature 508:351-356. CrossRef Medline

Fotowat H, Harrison RR, Gabbiani F (2011) Multiplexing of motor information in the discharge of a collision detecting neuron during escape behaviors. Neuron 69:147-158. CrossRef Medline

Gabbiani F, Krapp HG, Laurent G (1999) Computation of object approach by a wide-field, motion-sensitive neuron. J Neurosci 19:1122-1141. Medline

Gebhardt M, Honegger HW (2001) Physiological characterisation of antennal mechanosensory descending interneurons in an insect (Gryllus bimaculatus, Gryllus campestris) brain. J Exp Biol 204:2265-2275. Medline

Gentet LJ, Kremer Y, Taniguchi H, Huang ZJ, Staiger JF, Petersen CC (2012) Unique functional properties of somatostatin-expressing GABAergic neurons in mouse barrel cortex. Nat Neurosci 15:607-612. CrossRef Medline

Goldammer J (2013) Morphology of antennal hair field afferents, descending interneurons and mesothoracic motoneurons, and in situ calcium imaging of retrogradely labeled retractor coxae neurons in the stick insect. $\mathrm{PhD}$ thesis, Universität zu Köln.

Gonzalez-Bellido PT, Peng H, Yang J, Georgopoulos AP, Olberg RM (2013) Eight pairs of descending visual neurons in the dragonfly give wing motor centers accurate population vector of prey direction. Proc Natl Acad Sci U S A 110:696-701. CrossRef Medline

Guo P, Ritzmann RE (2013) Neural activity in the central complex of the cockroach brain is linked to turning behaviors. J Exp Biol 216:992-1002. CrossRef Medline

Harley CM, English BA, Ritzmann RE (2009) Characterization of obstacle negotiation behaviors in the cockroach, Blaberus discoidalis. J Exp Biol 212:1463-1476. CrossRef Medline

Hedwig B (1994) A cephalothoracic command system controls stridulation in the acridid grasshopper Omocestus viridulus L. J Neurophysiol 72: 2015-2025. Medline

Heinze S, Homberg U (2007) Maplike representation of celestial E-vector orientations in the brain of an insect. Science 315:995-997. CrossRef Medline

Hill PS (2009) How do animals use substrate-borne vibrations as an information source? Naturwissenschaften 96:1355-1371. CrossRef Medline

Hutson KA, Masterton RB (1986) The sensory contribution of a single vibrissa's cortical barrel. J Neurophysiol 56:1196-1223. Medline

Ito K, Shinomiya K, Ito M, Armstrong JD, Boyan G, Hartenstein V, Harzsch S, Heisenberg M, Homberg U, Jenett A, Keshishian H, Restifo LL, Rössler W, Simpson JH, Strausfeld NJ, Strauss R, Vosshall LB (2014) A systematic nomenclature for the insect brain. Neuron 81:755-765. CrossRef Medline

Knutsen PM, Pietr M, Ahissar E (2006) Haptic object localization in the vibrissal system: behavior and performance. J Neurosci 26:8451-8464. CrossRef Medline

Krause AF, Dürr V (2004) Tactile efficiency of insect antennae with two hinge joints. Biol Cybern 91:168-181. CrossRef Medline

Krause AF, Winkler A, Dürr V (2013) Central drive and proprioceptive control of antennal movements in the walking stick insect. J Physiol Paris 107:116-129. CrossRef Medline

Levine J, Tracey D (1973) Structure and function of the giant motorneuron of Drosophila melanogaster. J Comp Physiol 87:213-235. CrossRef

Matheson T (1992) Range fractionation in the locust metathoracic femoral chordotonal organ. J Comp Physiol A Neuroethol Sens Neural Behav Physiol 170:509-520.

Okada J, Toh Y (2000) The role of antennal hair plates in object-guided tactile orientation of the cockroach (Periplaneta americana). J Comp Physiol A Neuroethol Sens Neural Behav Physiol 186:849-857. CrossRef Medline

Okada J, Toh Y (2001) Peripheral representation of antennal orientation by the scapal hair plate of the cockroach Periplaneta americana. J Exp Biol 204:4301-4309. Medline

O'Shea M, Williams JLD (1974) The anatomy and output connection of a locust visual interneurone: the lobular giant movement detector (LGMD) neurone. J Comp Physiol 91:257-266. CrossRef

Petersen CC (2014) Cortical control of whisker movement. Annu Rev Neurosci 37:183-203. CrossRef Medline

Ritzmann RE, Ridgel AL, Pollack AJ (2008) Multi-unit recording of antennal mechano-sensitive units in the central complex of the cockroach, Blaberus discoidalis. J Comp Physiol A Neuroethol Sens Neural Behav Physiol 194:341-360. CrossRef Medline

Roeder KD (1937) The control of tonus and locomotor activity in the praying mantis (Mantis religiosa L.). J Exp Zool 76:353-374. CrossRef

Schöneich S, Schildberger K, Stevenson PA (2011) Neuronal organization of a fast-mediating cephalothoracic pathway for antennal-tactile information in the cricket (Gryllus bimaculatus DeGeer). J Comp Neurol 519: 1677-1690. CrossRef Medline

Schütz C, Dürr V (2011) Active tactile exploration for adaptive locomotion in the stick insect. Philos Trans R Soc Lond B Biol Sci 366:2996-3005. CrossRef Medline

Scott SH (2004) Optimal feedback control and the neural basis of volitional motor control. Nat Rev Neurosci 5:532-546. CrossRef Medline

Seelig JD, Jayaraman V (2013) Feature detection and orientation tuning in the Drosophila central complex. Nature 503:262-266. CrossRef Medline

Siegler MV, Burrows M (1979) The morphology of local non-spiking interneurones in the metathoracic ganglion of the locust. J Comp Neurol 183:121-147. CrossRef Medline

Staudacher E, Gebhardt M, Dürr V (2005) Antennal movements and mechanoreception: neurobiology of active tactile sensors. Adv Insect Physiol 32:49-205. CrossRef

Stengl M, Homberg U, Hildebrand JG (1990) Acetylcholinesterase activity in antennal receptor neurons of the sphinx moth Manduca sexta. Cell Tissue Res 262:245-252. CrossRef Medline

Tanouye MA, Wyman RJ (1980) Motor outputs of giant nerve fiber in Drosophila. J Neurophysiol 44:405-421. Medline

Theunissen LM, Vikram S, Dürr V (2014) Spatial coordination of foot contacts in unrestrained climbing insects. J Exp Biol 217:3242-3253. CrossRef Medline

von Reyn CR, Breads P, Peek MY, Zheng GZ, Williamson WR, Yee AL, Leonardo A, Card GM (2014) A spike-timing mechanism for action selection. Nat Neurosci 17:962-970. CrossRef Medline

Vincent SB (1912) The function of vibrissae in the behavior of the white rat. Behav Monogr 1:1-81.

Zurek DB, Gilbert C (2014) Static antennae act as locomotory guides that compensate for visual motion blur in a diurnal, keen-eyed predator. Proc Biol Sci 281:20133072. CrossRef Medline 